\title{
Interactive Fluid Animation and Its Applications
}

\author{
Jeongjin Lee ${ }^{1}$, Helen Hong ${ }^{2}$, and Yeong Gil Shin ${ }^{1}$ \\ ${ }^{1}$ School of Electrical Engineering and Computer Science, \\ Seoul National University; \\ \{jjlee, yshin\}@cglab.snu.ac.kr \\ ${ }^{2}$ School of Electrical Engineering and Computer Science BK21, \\ Information Technology, Seoul National University, \\ San 56-1 Shinlim 9-dong Kwanak-gu, Seoul 151-742, Korea \\ hlhong@cse.snu.ac.kr
}

\begin{abstract}
In this paper, we propose a novel technique of fluid animation for interactive applications. We have incorporated an enhanced particle dynamics simulation method with pre-integrated volume rendering. The particle dynamics simulation of fluid flow can be conducted in real-time using the Lennard-Jones model. The computational efficiency is enhanced since a small number of particles can represent a significant volume. To get a high-quality rendering image with small data, we use the pre-integrated volume rendering technique. Experimental results show that the proposed method can be successfully applied to various fluid animation applications at interactive speed with acceptable visual quality.
\end{abstract}

\section{Introduction}

The demand for interactive fluid animation has increased recently for $3 \mathrm{D}$ computer games and virtual reality applications. However, it is very difficult to animate natural fluid phenomena at interactive speed, because their motions are so complex and irregular that intensive simulation and rendering time is needed.

In the previous work, only off-line fluid animation methods have been reported [13]. In general, fluid animation is carried out by physical simulation immediately followed by visual rendering. For the physical simulation of fluids, the most frequently used practices are the particle dynamics simulation of isolated fluid particles and the continuum analysis of flow via the Navier-Stokes equation. Miller et al. [4] proposed a spring model among particles to represent viscous fluid flow. Terzopoulos et al. [5] introduced molecular dynamics to consider interactions between particles. In these approaches, when the number of particles increases significantly, the number of related links between particles exponentially increases. Therefore, it takes too much time for realistic fluid simulation due to the large number of particles for describing complex fluid motions. Stam [1] proposed a precise and stable method to solve the Navier-Stokes equations for any time step. Foster [3] applied a 3D incompressible Navier-Stokes equation. Above methods using the Navier-Stokes equations yield a realistic fluid motion when properly conditioned, but still need huge calculations of complex equations. The second limitation is the time 
complexity of visual rendering. Global illumination has been widely used for natural fluid animation. Jensen et al. [6] proposed a photon mapping method currently used in many applications. Global illumination is generally successful in rendering premiumquality images, but too slow to be used in interactive applications.

In this paper, we propose a novel technique for interactive fluid animation and its applications. For rapid analysis of the motion of fluids, we use a modified form of particle dynamic equations. The fluid interaction is approximated by the attractive and repulsive forces between adjacent particles using the Lennard-Jones model to emulate fluid viscosity. To get a high quality rendering image with a smaller volume data, we use a pre-integrated volume rendering method [7]. Experimental results show that our method is successfully applied to various interactive fluid animation applications.

The organization of the paper is as follows. In Section 2, we discuss the particle dynamics simulation of our method, and describe how the simulation data are rendered. In Section 3, experimental results show various kinds of interactive fluid animation applications. This paper is concluded with brief discussions of the results in Section 4.

\section{Interactive Fluid Animation Methodology}

\subsection{Dynamics Simulation of Fluid Particles}

Two approaches, particle dynamics and continuum dynamics, have been widely used for fluid simulation. The continuum dynamics approach is not suitable for interactive applications due to its high time complexity of calculating the Navier-Stokes equation [1-3]. In our approach, a simple particle dynamics approach is chosen since it is much faster than a continuum dynamics approach based on the Navier-Stokes equation.

In particle dynamics, a spherical particle is assumed to be the basic element that makes an object such as for solid, liquid and gas, and used for calculating interactions between particles. For $\mathrm{N}$ spherically symmetric particles, the total inter-particle potential energy $E\left(\mathbf{r}^{N}\right)$ is the sum of isolated pair interactions according to pair-wise addition.

$$
E\left(\mathbf{r}^{N}\right)=\sum_{i=1}^{N} \sum_{j=1}^{N} u\left(r_{i j}\right), i \neq j,
$$

where $\mathbf{r}^{N}$ is the set of vectors that locate centers of mass, i.e. $\mathbf{r}^{N}=\left\{\mathbf{r}_{1}, \mathbf{r}_{2}, \mathbf{r}_{3}, \ldots, \mathbf{r}_{N}\right\}$ and $r_{i j}$ is the scalar distance between particles $\mathrm{i}$ and $\mathrm{j}$.

The elementary potential energy $u\left(r_{i j}\right)$ is taken from the Lennard-Jones (LJ) potential model [8]. For two particles i and $\mathrm{j}$ separated by a distance $r_{i j}$, the potential energy $u\left(r_{i j}\right)$ between the both can be defined as

$$
u\left(r_{i j}\right)=4 \varepsilon\left(\left(\frac{\sigma}{r_{i j}}\right)^{12}-\left(\frac{\sigma}{r_{i j}}\right)^{6}\right) .
$$


The force field $f_{i j}$ created by two particles $\mathrm{i}$ and $\mathrm{j}$ can be given as

$$
f_{i j}=-\frac{d u\left(r_{i j}\right)}{d r_{i j}}=\left(\frac{48 \varepsilon}{\sigma^{2}}\right)\left(\left(\frac{\sigma}{r_{i j}}\right)^{14}-\frac{1}{2}\left(\frac{\sigma}{r_{i j}}\right)^{8}\right) r_{i j}
$$

Since the inter-particle potential forces are conservative within a given potential field, the overall potential force $F_{i, p}$ acting on particle $\mathrm{i}$ is related to the potential by

$$
F_{i, p}=-\frac{\partial E\left(\mathbf{r}^{N}\right)}{\partial \mathbf{r}_{i}}=m_{i} \ddot{\mathbf{r}}_{i}
$$

where $m_{i}$ is the mass of particle i. Fig. 1 illustrates the Lennard-Jones potential and the force extended over a modest range of pair separations. The critical distance at which the positive sign of the inter-particle force becomes negative can be considered as the particle radius.

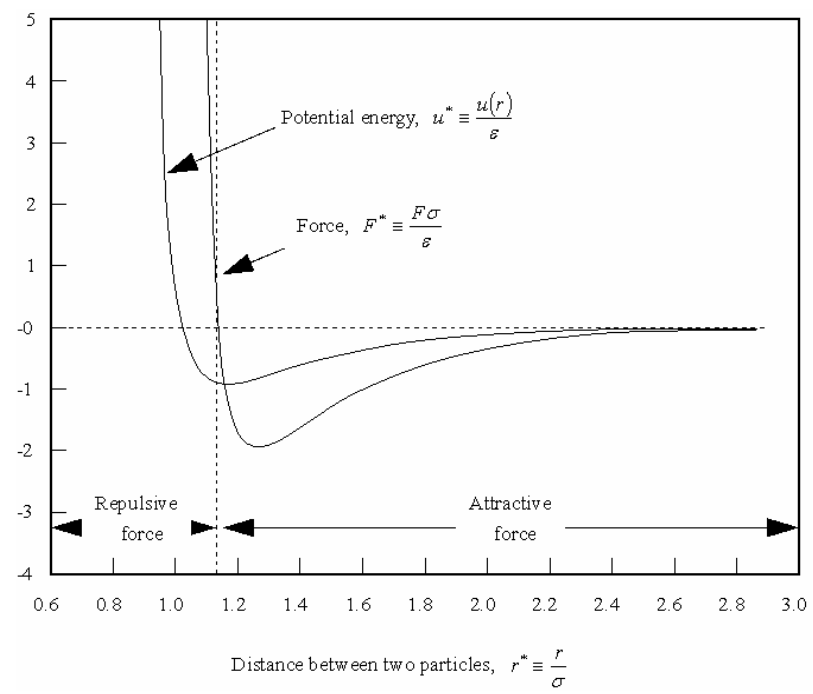

Fig. 1. The Lennard-Jones potential and the force

The friction force $F_{i, f}$ on particle i can be given as

$$
F_{i, f}=-\zeta \dot{\mathbf{r}}_{i},
$$

where $\zeta$ is the friction coefficient. The governing equation of the force balance on particle $\mathrm{i}$ can hence be written as 


$$
-\frac{\partial E\left(\mathbf{r}^{N}\right)}{\partial \mathbf{r}_{i}}+\zeta \dot{\mathbf{r}}_{i}=0 .
$$

Eq. (6) can be used to calculate the interaction between particles by assuming them as slightly deformable soft spheres. The soft sphere collision method is useful for treating interactions between particles with the separation distance within a moderate multiple of the critical distance.

\subsection{Realistic Rendering of Simulation Data}

A photon mapping method [6] accomplishes the global illumination effect using ray tracing, which is excessively slow for interactive applications. For interactive rendering without the loss of image quality, we use a pre-integrated volume rendering on graphics hardware. Rendering of simulation data is accomplished in the following three steps. In the first step, we transform the simulation data into volume data. We divide the 3D space, in which particles of simulation data are stored, into regular cells having unit length $\mathrm{d}$. The density of each cell is determined by the volume fraction value as Eq. (7). These density values are used for volume rendering.

$$
\text { Volume fraction }=\frac{n \times \frac{4}{3} \pi r^{3}}{d^{3}},
$$

where $\mathrm{n}$ is the number of particles in the cell and $\mathrm{r}$ is the radius of particles. In the second step, we visualize volume data using a pre-integrated volume rendering technique. The color and opacity between two neighboring slices of volume data are pre-integrated and stored in the graphics hardware texture memory for acceleration. Using the pre-integrated volume rendering technique accelerated by graphics hardware we can get the high quality image with a smaller volume data at the interactive rate. In the third step, we can control the opacity transfer function, which assigns different colors and opacity values according to volume data. Various visual effects can be generated by interactively modifying the opacity transfer function.

\section{Experimental Results}

All of the implementations have been performed on an Intel Pentium IV PC containing $2.4 \mathrm{GHz}$ CPU with GeForce FX 5800 graphics hardware. Fig. 2 and 3 show the animation of water flowing from a bottle to a cup. The opacity transfer function of this animation is given in Table 1. Between two control points $\mathrm{Vi}$ and $\mathrm{Vj}$, color and opacity are determined using linear interpolation. As shown in Fig. 2, when the number of particles (n) is 2000 and the radius of particles (r) is 0.002 [m], particle characteristics of the water are emphasized. This animation occurs in $7 \sim 8 \mathrm{fps}$ at 730 x 520 resolution. Fig. 3 shows that when the number of particles (n) is 10000 and the radius of particles (r) is 0.003 [m], continuum characteristics of the water are emphasized. This animation occurs in $3 \sim 4 \mathrm{fps}$ at $730 \times 520$ resolution. 
Table 1. Opacity transfer function for water animation

\begin{tabular}{|l|l|}
\hline $\mathrm{d} 1 / \mathrm{V} 1(\mathrm{R}, \mathrm{G}, \mathrm{B}, \mathrm{A})$ & $0.21 /(0,0,255,0.0)$ \\
\hline $\mathrm{d} 2 / \mathrm{V} 2(\mathrm{R}, \mathrm{G}, \mathrm{B}, \mathrm{A})$ & $0.23 /(36,65,91,1.0)$ \\
\hline $\mathrm{d} 3 / \mathrm{V} 3(\mathrm{R}, \mathrm{G}, \mathrm{B}, \mathrm{A})$ & $0.28 /(154,208,228,1.0)$ \\
\hline $\mathrm{d} 4 / \mathrm{V} 4(\mathrm{R}, \mathrm{G}, \mathrm{B}, \mathrm{A})$ & $0.30 /(0,0,160,0.0)$ \\
\hline
\end{tabular}

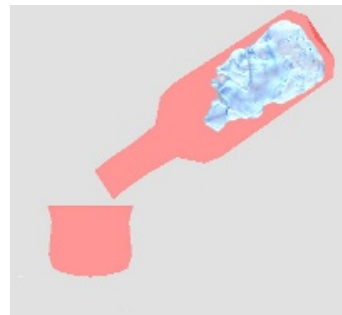

(a)

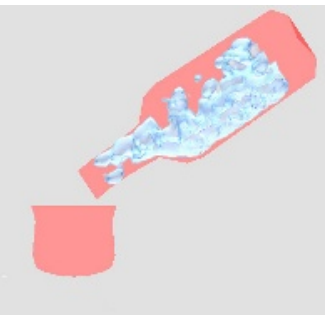

(b)

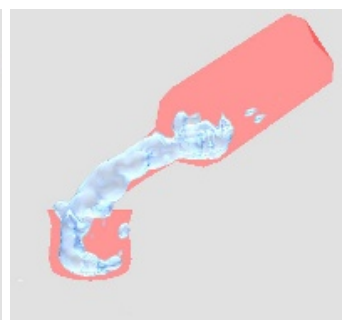

(c)

Fig. 2. The animation of pouring water from a bottle into a cup $(n=2000, r=0.002[\mathrm{~m}])$ (a) $t=$ $0.12[\mathrm{~s}](\mathrm{b}) \mathrm{t}=0.33[\mathrm{~s}](\mathrm{c}) \mathrm{t}=1.02[\mathrm{~s}]$

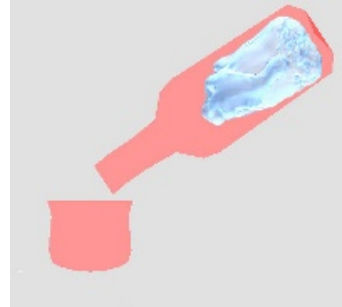

(a)

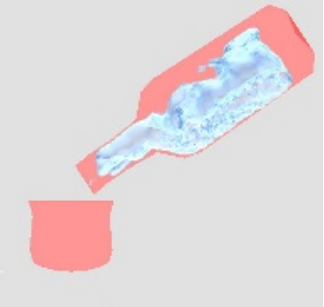

(b)

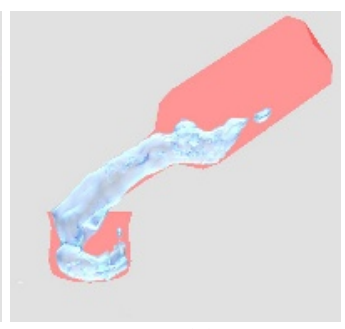

(c)

Fig. 3. The animation of pouring water from a bottle into a cup $(n=10000, r=0.003[\mathrm{~m}])$ (a) $t$ $=0.12[\mathrm{~s}](\mathrm{b}) \mathrm{t}=0.33[\mathrm{~s}](\mathrm{c}) \mathrm{t}=1.02[\mathrm{~s}]$

Fig. 4 shows the comparison of our method with RealFlow, which is the widely used commercial software for fluid animation. The average number of particles used for the animation of RealFlow is 1870000 while that of our method is 8000 . For the generation of 10 seconds' animation in Fig. 4(e), (f), RealFlow computes during 4 hours 30 minutes whereas our method finishes within 150 seconds. The experimental results show that our method gives similar visual quality comparing with RealFlow using much smaller number of particles. In addition, total processing time of our method is dramatically faster than that of RealFlow.

Fig. 5 shows water and artificial fluid flow using our fluid animation method. Fig. 6(a) shows a conceptual overview of fluidic shadow dance application. The shadow of the viewer is projected onto a screen by a light. Two columns to the left and right of the viewer are equipped with a vertical array of switches. When the viewer's hand 


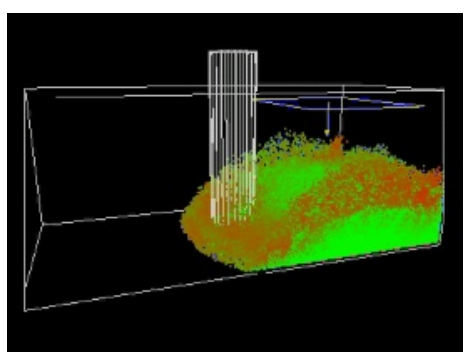

(a)

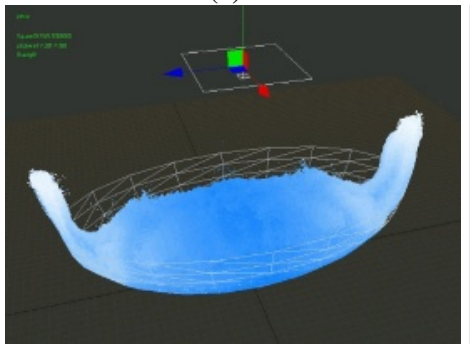

(c)

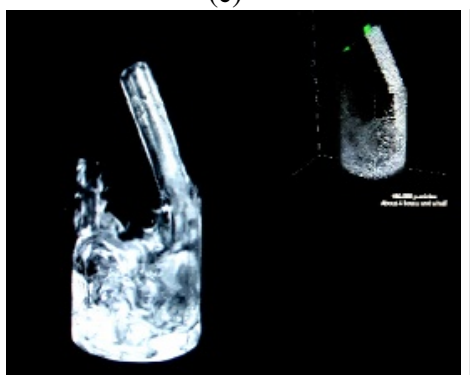

(e)

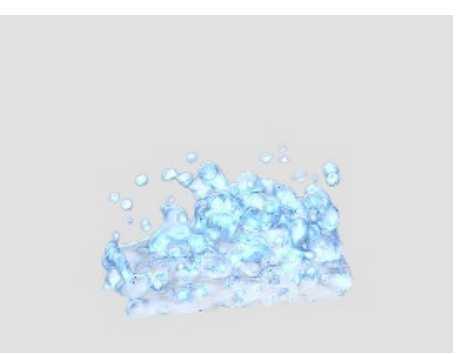

(b)

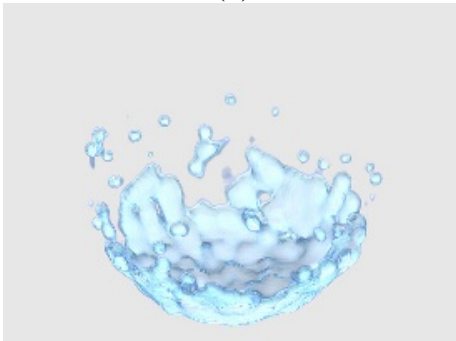

(d)

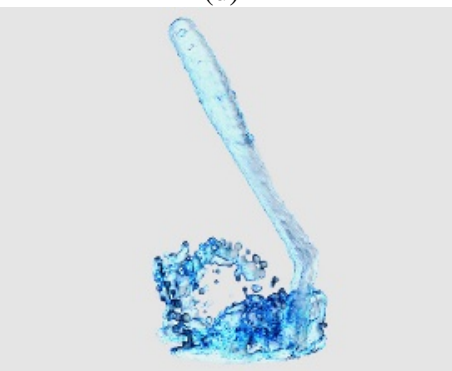

(f)

Fig. 4. Comparison of our method with RealFlow (a) RealFlow ( $\mathrm{n}=160000)$ (b) our method ( $\mathrm{n}$ $=8000)(\mathrm{c})$ RealFlow $(\mathrm{n}=5000000)(\mathrm{d})$ our method $(\mathrm{n}=6000)(\mathrm{e})$ RealFlow $(\mathrm{n}=450000)(\mathrm{f})$ our method $(\mathrm{n}=10000)$

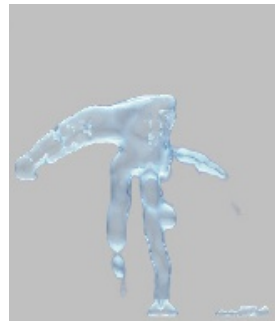

(a)

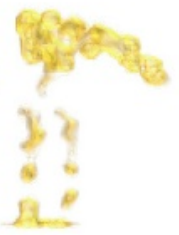

(b)

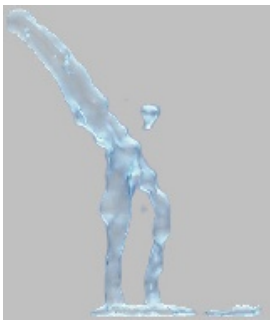

(c)

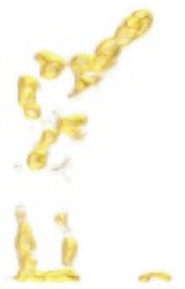

(d)

Fig. 5. Fluid animation for fluidic shadow dance application (a) water flow from left-lower side (b) artificial fluid flow from right-lower side (c) water flow from left-upper side (d) artificial fluid flow from right-upper side 


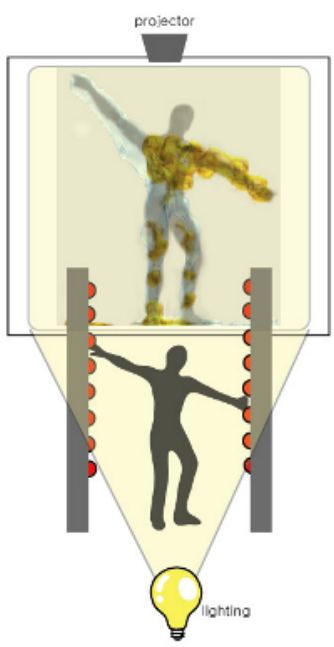

(a)

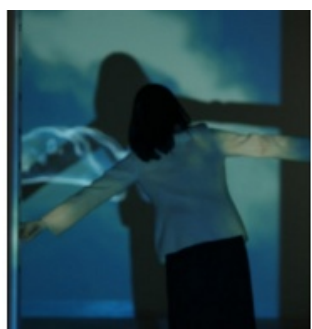

(b)

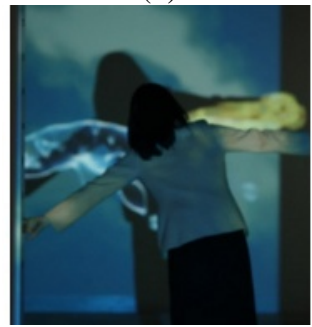

(c)

Fig. 6. The fluidic shadow dance application (a) conceptual overview (b) flow from left column (c) flow from both columns

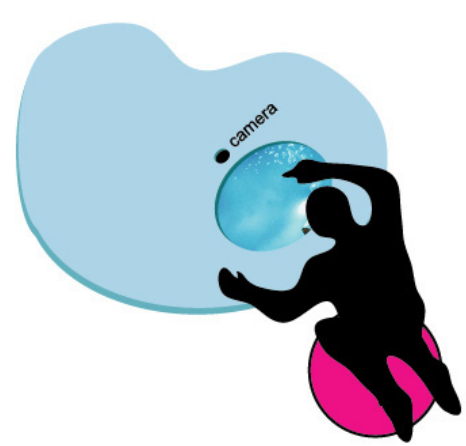

(a)

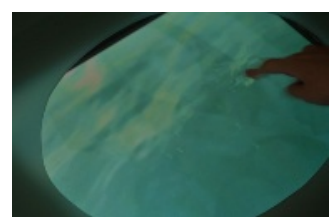

(b)

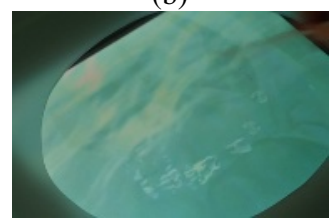

(c)

Fig. 7. The fluid portrait application (a) conceptual overview (b) by fingertip (c) by stick

comes in contact with a specific location, the corresponding switch is inputted into our program, and the image of a fluid flowing out from that location is animated. Fluids coming out of the two columns are ejected towards the center of the viewer's body, subsequently fall towards the bottom simulating gravity, collide into one another, and then splash upwards resulting in a free and irregular motion. The demonstration of this interactive application is shown in Fig. 6(b), (c).

Fig. 7(a) shows a conceptual overview of fluid portrait application. A camera projects the viewer's face onto the surface of a touch screen. Interaction is created between the viewer's hand movements and rendered fluid in real-time. If the viewer 
continuously stimulates a certain spot, small water droplets merge together to form a large water drop. When the viewer stimulates a different spot, the merged water drop disperses and moves to the other stimulated spot to form into a large water drop again. The demonstration of this interactive application is shown in Fig. 7(b), (c).

\section{Conclusion}

This paper presents a novel technique of fluid animation, which integrates particle dynamics simulation and pre-integrated volume rendering. The particle dynamics simulation can be conducted in real-time using the Lennard-Jones model. Furthermore, pre-integrated volume rendering allowed us to avoid the unnaturalness of images usually obtained with particle dynamics, and achieve an image quality good enough for interactive applications. In the animation of water, both the particle and continuum characteristics of the water can be realistically displayed by manipulating simulation parameters. Comparing with widely used commercial software, our fluid animation method is performed at dramatically faster speed with comparable visual quality. Various experimental results show that our method can be successfully applied to interactive fluid animation applications.

\section{References}

1. Stam, J., Stable Fluids, ACM SIGGRAPH (1999) 121-128.

2. Foster, N., Practical Animation of Liquids, ACM SIGGRAPH (2001) 23-30.

3. Foster, N., Realistic Animation of Liquids, Graphical Models and Image Processing (1996) 204-212.

4. Miller, Gavin S. P., Pearce, A., Globular Dynamics: A Connected Particle System for Animating Viscous Fluids, Computers and Graphics Vol. 13, No. 3 (1989) 305-309.

5. Terzopoulos, Platt, Fleischer, From Goop to Glop: Melting Deformable Models, Graphics Interface (1989).

6. Jensen, H.W., Christensen, P.H., Efficient Simulation of Light Transport in Scenes with Participating Media using Photon Maps, ACM SIGGRAPH (1998) 311-320.

7. Engel, K., Kraus, M., Ertl, T., High-Quality Pre-Integrated Volume Rendering Using Hardware-Accelerated Pixel Shading, Siggraph/Eurographics Workshop on Graphics Hardware (2001) 9-16.

8. McQuarrie, D.A., Statistical Mechanics, Harper and Row (1976). 